\title{
Niosomes as Carrier in Dermal Drug Delivery
}

\author{
Yahya Rahimpour and Hamed Hamishehkar
}

Additional information is available at the end of the chapter

http://dx.doi.org/10.5772/51729

\section{Introduction}

Colloidal vesicular carriers such as liposomes or niosomes have been extensively applied in drug delivery systems due to unique advantages. These vesicles can act as drug reservoirs and the rate of drug release can be modified by changing of their composition. These lipid carriers can encapsulate both hydrophilic drugs (by loading in inner space) and hydrophobic drugs (in lipid area). Because of their potential to carry a variety of drugs, these lipid vesicles have been widely used in various drug delivery systems like drug targeting, controlled release and permeation enhancement of drugs (Akhilesh et al., 2011). Dermal (topical) delivery defines a targeting to the pathological sites within the skin with the least systemic absorption. Drug localization in this case is a crucial issue in the treatment of dermatological problems such as skin cancer, psoriasis, alopecia and acne, where the origin of disease is located in the skin (Brown et al., 2006). Topical drug administration has been initiated since long time to accomplish several functions on different skin levels (skin surface, epidermis, dermis and hypodermis). But, several limitations have been associated with the conventional topical preparations e.g. low percutaneous penetration because of the barrier function of the stratum corneum, the outermost layer of the skin, (Rubio et al., 2011) and absorption to the systemic circulation (Dubey et al., 2012). The scientific reports nowadays offer several systems that can be able to deliver drugs through the skin (Higaki et al., 2005). Recently niosomes are becoming popular in the field of topical drug delivery due to its outstanding characteristics like enhancing the penetration of drugs, providing a sustained pattern of drug release and ability to carry both hydrophilic and lipophilic drugs (Sathali et al., 2010). This chapter deals with the potential of niosome in topical delivery system focusing on its clinical approach. Skin represents a multilayered effective barricade to the penetration of drugs. The outer layer of skin, stratum corneum, provides a rate limiting step during percutaneous absorption of drug. Drug transfer across the stratum corneum is mainly a passive process, and thus the physicochemical properties of a permeant have a key role in its capability to penetrate and diffuse across the membrane. Compounds can penetrate 
through the stratum corneum via three routes: intercellular, transcellular (paracellular), and transappendageal (figure 1). Once it has transferred through the epidermis, a compound may be carried away by the dermal blood circulation or to be transported to deeper tissues. The relative significance of these penetration pathways will be largely dependent on the physicochemical characteristics of the drug molecules, particularly the partition and diffusion coefficients into the protein or lipid regions (Barry, 1991).

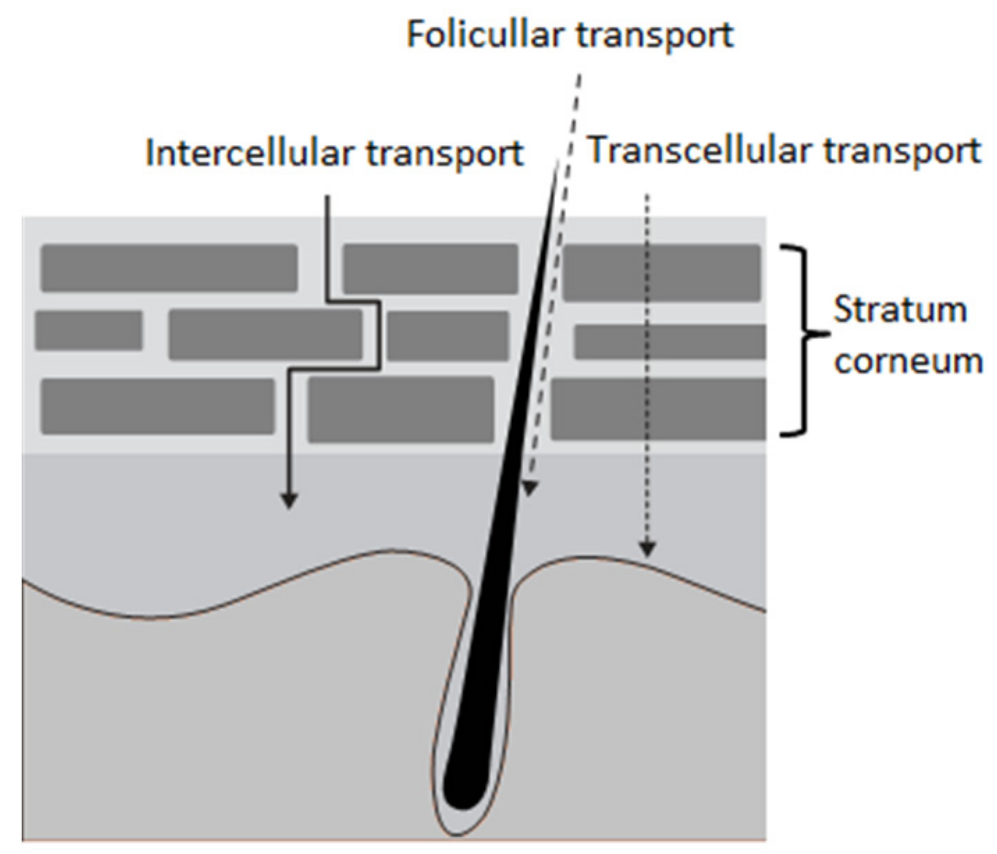

Figure 1. Possible pathways for delivery of compounds across the stratum corneum adapted from reference (Gašperlin et al., 2011).

For successful dermal or transdermal delivery, a reversible overcoming of skin barrier is required. Therefore, many strategies have been assessed to overcome the barrier function of the stratum corneum and to improve drug transport into the skin (Ghafourian et al., 2004; Rahimpour et al., 2012). Targeting of topically administered drugs to the different skin layers and appendages is becoming a main center of interest for many pharmaceutical research groups studying in dermatology (Nounou et al., 2008). The carrier is one of the most important entities required for successful targeted drug delivery (figure 2). Carriers will enable a drug to reach the desired pharmacological site of action at a controlled rate and to have a sustained duration of action. Consequently, as a vehicle for active substances and targeting to skin layers, surfactant based carriers such as niosomal systems are gaining more interest. 


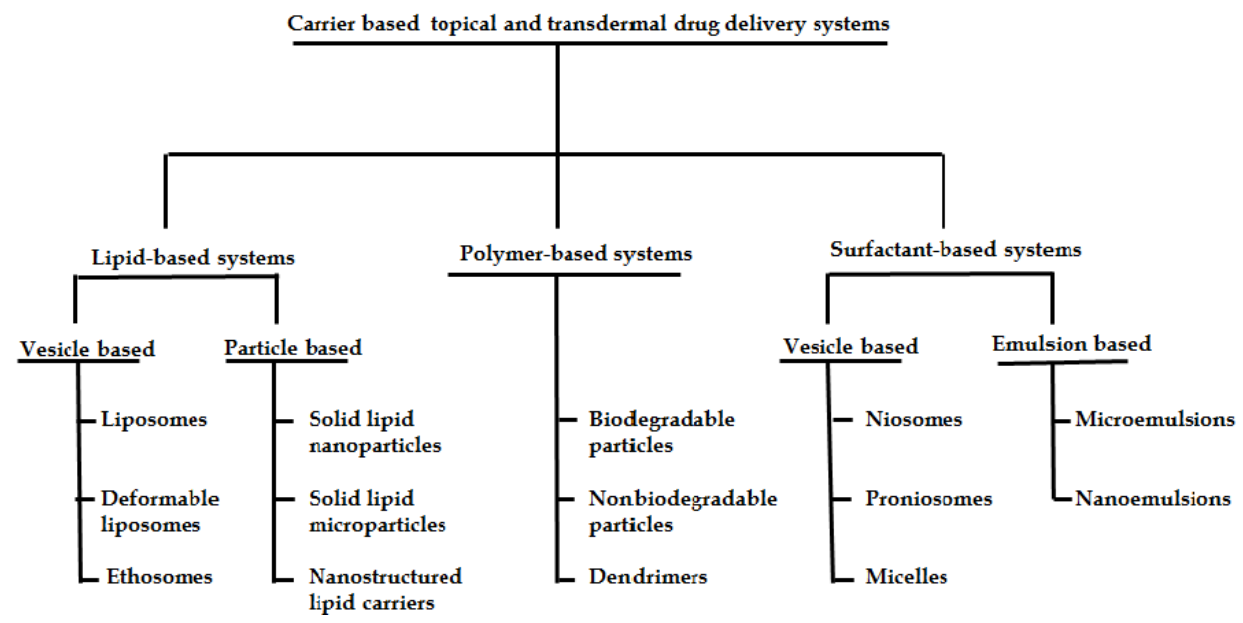

Figure 2. Carriers for topical and transdermal drug delivery adapted from reference (Venuganti et al., 2009).

\section{Niosome}

Niosomes are microscopic lamellar structures composed of non-ionic surfactants and cholesterol. The niosomes have amphiphillic bilayer structure in a way that polar region is oriented outside and inside the vesicles where the hydrophilic drug will be entrapped and nonpolar region is formed within the bilayer where hydrophobic drug can be entrapped (Khan et al., 2011). The formation of vesicular system based on hydration of mixture of a single-alkyl chain nonionic surfactant and cholesterol was firstly reported in 1979 (Handjani-Vila et al., 1979). Niosomes might be produced by various types of nonionic surfactants including polyglycerol alkyl ethers, crown ethers, ester-linked surfactants, glucosyldialkyl ethers, polyoxyethylene alkyl ethers, Brij, Tweens and Spans. Nonionic surfactants used to prepare niosomes carry no charge and are relatively nontoxic and mild to use (Azeem et al., 2009).

\subsection{Types of niosomes}

\subsubsection{Proniosomes}

Proniosome is a dry granular product that can be able to form a niosome suspension after hydration (Hu et al., 2000). Proniosomes are developed to overcome the disadvantage of vesicular system. Proniosome are prepared based on the simple scheme that a mixture of surfactant/alcohol/aqueous phase can be used to prepare a concentrated proniosome gel, which can instinctively be converted to a stable niosomal dispersion by dilution with excess aqueous phase (Perrett et al., 1991).

$$
\begin{aligned}
& \text { Carrier }+ \text { surfactant }=\text { Proniosomes } \\
& \text { Proniosomes }+ \text { water }=\text { Niosomes }
\end{aligned}
$$




\subsubsection{Aspasomes}

Mixture of acorbyl palmitate, cholesterol and highly charged lipid diacetyl phosphate leads to the construction of vesicles named aspasomes. Aspasomes are first hydrated with water/aqueous solution and then sonicated to attain the niosomes. Aspasomes are suggested to improve the transdermal permeation of drugs. Aspasomes have also been used to reduce disorders caused by reactive oxygen species due to its intrinsic antioxidant property (Gopinath et al., 2004; Rajera et al., 2011).

\subsubsection{Vesicles in water and oil system (v/w/o)}

In this system suspension of aqueous niosomes $(\mathrm{v} / \mathrm{w})$ are emulsified into the oily phase at $60^{\circ} \mathrm{C}$ to form vesicle in water in oil emulsion (v/w/o) (Yoshida et al., 1992; Hu et al., 2000). Cooling to room temperature forms vesicle in water in oil gel (v/w/o gel) (Yoshioka et al., 1992). The prepared v/w/o gel can entrap the hydrophilic active ingredients which are susceptible for enzymatic degradation such as proteins/proteinous drugs and also provide a controlled release pattern in drug delivery.

\subsubsection{Deformable niosomes}

Elastic niosomes are prepared of nonionic surfactants, ethanol and water. They show superior to conventional niosomes due to their capability to increase penetration efficiency of a compound through intact skin by passing through pores in the stratum corneum, which are smaller than the vesicles (figure 3). The flexibility of their structure allows them to pass through pores that are less than one-tenth of these vesicles (Cevc, 1996; Cevc et al., 1996).

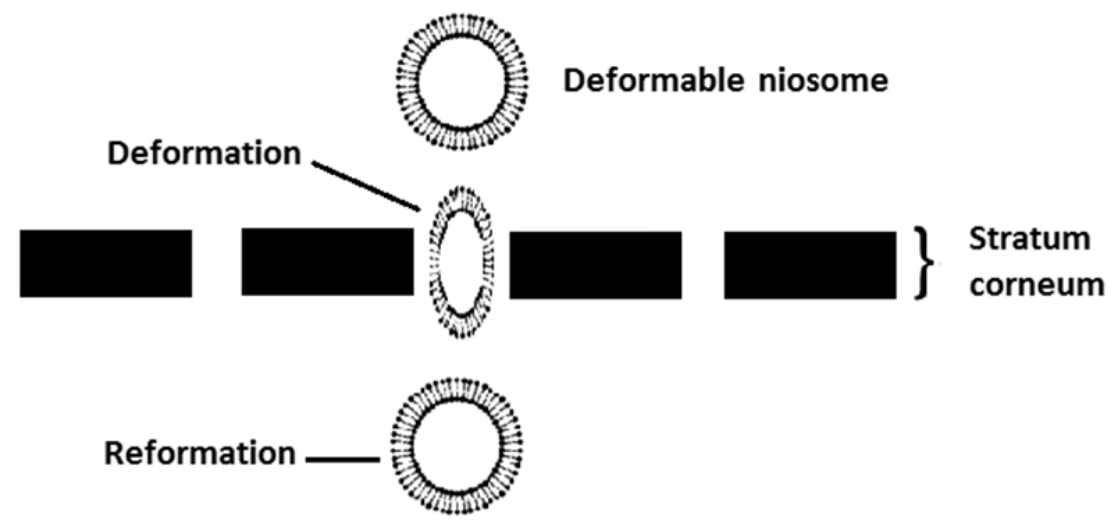

Figure 3. Mechanism of skin permeation of deformable niosome adapted from reference (Kumar et al., 2012)

\subsection{Advantages of noisome}

The major advantages of niosomes can be mentioned as: i) niosomes are biodegradable, biocompatible, non-toxic and non-immunogenic (Azeem et al., 2009); ii) niosomes are able to 
encapsulate large amount of materials in a small volume of vesicles (Nasr et al., 2008); iii) niosomes have better patient adherence and satisfication and also better effectiveness than conventional oily formulations (Jain et al., 2006); iv) niosomes can entrap wide range of chemicals (hydrophilic, lipophilic and amphiphilic drugs) due to its unique structure (Raja Naresh et al., 1994); v) the characteristics of niosome such as shape, fluidity and size can be easily controlled by changing in structural composition and method of production (Bayindir et al., 2010); vi) niosomes can be prescribed via different administration routes such as oral, parenteral and topical, etc. with different dosage forms such as semisolids, powders, suspensions (Malhotra et al., 1994) and vii) due to chemical stability of structural composition, the storage of the niosome is easy (Jain et al., 2006). In spite of several above mentioned merits, niosomes show some disadvantages. The aqueous suspensions of niosomes may undergo fusion, aggregation, leaking of entrapped drugs, and hydrolysis of encapsulated drugs, which lead to limited shelf life. The methods of preparation of multilamellar vesicles such as extrusion, sonication, are time consuming and may require specialized equipments for processing (Khan et al., 2011)

\subsection{Application of niosome}

Niosomes have been successfully used in drug targeting to various organs such as skin (Agarwal et al., 2001), brain (Abdelkader et al., 2012), liver (Baillie et al., 1986; Lala et al., 2004), lung (Desai et al., 2002; Abd-Elbary et al., 2008) ocular systems (Pugeat et al., 1991; Abdelkader et al., 2012), tumor (Ruckmani et al., 2000; Shi et al., 2005) etc. Niosomes show a higher bioavailability than conventional dosage forms (Raja Naresh et al., 1994). Controlled and sustained release of drugs have been achieved by niosomes (Bayindir et al., 2010). Permeation of drugs through the skin has been enhanced by niosomes (Pardakhty et al., 2007). Noisome, itself improves the stratum corneum properties both by reducing transepidermal water loss and skin condition by increasing smoothness via reloading lost skin lipids (Firthouse et al.). Niosomes can be applied for drug protection from biological enzymes and acid thereby increasing the drug stability (Jain et al., 2006).

\subsection{Niosomes in comparison with liposomes}

Niosomes and liposomes have similar application in drug delivery but chemically differ in structure units. Niosomes constitute of non-ionic surfactant whereas liposomes comprise of phospholipids (Khan et al., 2011). They are functionally the same, have the same physical properties and act as amphiphilic vesicules. Both can be used in targeted and sustained drug delivery system. Property of both depends upon composition of the bilayer and methods of their preparation (Verma et al., 2012). Studies have also shown that the function of niosomes in vivo is similar to that of liposomes (Hofland et al., 1994). In spite of these comparable characteristics, niosomes offer several advantages over liposomes: intrinsic skin penetration enhancing properties, higher chemical stability and lower costs (Nasr et al., 2008). Both of the last features make the niosome more attractive for industrial manufacturing ( $\mathrm{Hu}$ et al., 2000). Also, niosomes do not require special conditions such as low temperature or inert atmosphere during preparation and storage (Verma et al., 2012). Although the niosome 
shows better chemical stability, the physical instability during dispersion may be comparable to that of the liposome. Both niosomes and liposomes are at risk of aggregation, fusion, drug leakage, or hydrolysis of entrapped drugs during storage (Hu et al., 2000; Rahimpour et al., 2012).

\section{Mechanisms of niosomal skin delivery}

Several mechanisms have been suggested to describe the ability of niosomes in transdermal and dermal drug delivery: i) niosomes diffuse from the stratum corneum layer of skin as a whole; ii) new smaller vesicles are formed in skin (re-formation of noisome vesicles). The water content of skin is crucial issue for interpreting and establishing this mechanism. Smaller diameter of lipid lamellar spaces of the stratum corneum than noisome vesicles makes this mechanism more meaningful (Sahin, 2007). iii) noisomes interact with stratum corneum with aggregation, fusion and adhesion to the cell surface which causes a high thermodynamic activity gradient of the drug at the vesicle-stratum corneum surface, which is the driving force for the penetration of lipophilic drugs across the stratum corneum (Ogiso et al., 1996). Scanning electron microscopy confirmed the fusion of niosome vesicles of estradiol on the surface of skin (Schreier et al., 1994) iv) niosomes may modify stratum corneum structure which makes the intercellular lipid barrier of stratum corneum looser and more permeable (Fang et al., 2001); v) non-ionic surfactant itself, the composing ingredient of niosome, acts as a permeation enhancer and might partly contribute to the improvement of drug permeation from niosomes (Javadzadeh et al., 2010). The type of surfactant plays an important role in modification of permeation using niosome vehicles. Niosomes fabricated from polyoxyethylene stearyl ether and existing in the gel state did not enhance estradiol permeation, and those prepared from polyoxyethylene lauryl ether and polyoxyethylene oleyl ether, both existing as liquid crystalline vesicles, considerably improved transport (Hofland et al., 1991).

\section{Toxicity of niosomes}

Surfactants are suspected to show toxicity but there are virtually not enough research about toxicity of niosomes (Yadav et al., 2011). A study on the toxicity effect of surfactant type of niosomal formulations on human keratocytes showed that the ester type surfactants are less toxic than ether type due to enzymatic degradation of ester bounds (Hofland et al., 1991). Hofland et al. studied the toxicity of CxEOy surfactants via ciliotoxicity model on nasal mucosa (which is important for intranasal administration) and on human kerationocytes (which is important for the transdermal application of vesicles). The results showed that increase in alkyl chain length of surfactant causes a reduction in toxicity, while, increase in the polyoxyethylne chain length enhances ciliotoxicity. The study supposed that ciliotoxicity related to liquid state formation with increasing in polyoxyethylene chain length, while increase in alkyl chain length of surfactant leads to formation of gel which is more safe than liquid state (Hofland et al., 1992). In another study, vincristine, a potent anti-tumor agent, was loaded into niosomes and administered intravenously. Result showed a significant 
increase in vincristine anti-tumor activity in S-180 sarcoma and Erlich ascites mouse models followed by reduction of its side effect such as diarrhea, neurological toxicity and alopecia compared with free drug (Parthasarathi et al., 1994). Toxicity effect of niosomes should be considered according to the intended route of delivery. For example, hemocompatibility studies should be carried out when the niosomes are meant to be delivered by intravenous route to evaluate their toxic potential (Uchegbu et al., 1997). The first in vivo experiment on drug delivery by means of synthetic non-ionic surfactant vesicles were carried out by Azmin et al. and reported that no unfavorable effects were observed in the performed experiment (Azmin et al., 1985). Rogerson et al. reported in vivo experiment over 70 male BALB/C mice and stated that no fatalities were related to niosomes. The drug associated toxicity were also reduced (Rogerson et al., 1987). Niosomes which have been prepared with Bola-surfactants showed a certain and encouraging safety and tolerability both in vitro on human keratinocyte cells up to an incubation time of $72 \mathrm{~h}$ for the different concentrations studied $(0.01-10 \mu \mathrm{M})$ and in vivo on human volunteers that showed no skin erythema when topically treated with the drug free Bola-niosome formulation (Paolino et al., 2007).

\section{Indications for niosomes as drug carriers in dermatology}

Niosomes were formulated and patented for the dermatological purposes in 1975 for the first time and since then many of products were developed based on this technology and appeared in the market such as Lancome Noisome Plus as an anti-ageing formulation (Azeem et al., 2009). Recently, the topical delivery of certain drugs using niosome has been developed. Here, current attempts with a focus on clinical application were reviewed to prove the ability of niosomes as the superior topical carrier.

\subsection{Local anesthesia}

Dermatologists take benefit of topical anesthetics for decreasing pain relief prior to cutaneous procedures, pain associated with laser pulses, or soft tissue augmentation. Inefficient formulations of local anesthetics resulted in severe dermatitis, systemic toxicity, or inadequate local analgesia. Carafa et al. fabricated lidocaine and lidocaine hydrochlorideloaded non-ionic surfactant vesicles using Tween $20^{\mathrm{TM}}$ and cholesterol. The ability of drug to diffuse through a model lipophilic membrane (Silastic ${ }^{\mathrm{TM}}$ ) and through mouse skin were studied and compared with classical liposomes and Tween 20 micelles. Dicetylphosphate and N-cetylpyridinium chloride were also used to prepare negatively and positively charged vesicles, respectively to study the effect of vesicle charge on drug encapsulation efficiency. Diffusion experiments indicated that the flux of charged lidocaine (lidocaine hydrochloride) through Silastic ${ }^{\mathrm{TM}}$ membrane was possible only after the vesicle encapsulation. Permeation of lidocaine hydrochloride-loaded vesicles through mouse abdominal skin presented a higher flux and a shorter lag time with respect to classical liposome formulations, whereas lidocaine permeation rate was quite similar for niosomes and liposome formulations. Furthermore fluorescence quenching study showed that positive and negative charged vesicles had negligible entrapment efficiency of drug, at $\mathrm{pH}$ 
5.5 compare with neutral vesicles (Tween 20 and cholesterol). The importance of non-ionic surfactant vesicles in dermal delivery of charged local anesthetics is concluded in this study (Carafa et al., 2002).

\subsection{Psoriasis}

Psoriasis is a chronic inflammatory condition of the skin which may drastically undermined the patient quality of life. Psoriasis, a T-lymphocyte-mediated autoimmune disease of the dermis and epidermis, is characterized by leukocyte infiltration into the skin and localized deregulated skin growth, which leads to the development of scaling erythematous plaques (Dubey et al., 2007). Although psoriasis is rarely life-threatening, it causes an unpleasant appearance that makes the patients to miss their confidence and suffer from itching, painful and disfiguring skin lesions. Methotrexate, anthralin, corticosteroids, coal tar, vitamin D3 analogs, tacrolimus and retinoids are administered topically for psoriasis treatment (Su et al., 2008). Topical therapy is the commonly used in patients, even though the use of topical formulations based on conventional excipients shows some disadvantages that limit considerably their use in therapy (Puglia et al., 2012). With the use of niosomes as carrier for topical drug delivery, the possibility to improve efficacy and safety of the topical products has improved manifold. Dithranol, one of the key medicines in the topical treatment of psoriasis, has staining, burning, irritating and necrotizing effects on the normal and also the diseased skin. Entrapment of dithranol in niosomal and liposomal systems could be achieved after optimizing the various process and formulation variables. These systems presented size stability and improved drug permeation properties. Although the in vitro study using laca mice abdominal skin shows higher skin penetration for vesicular systems compared with the cream base but niosomal vesicular systems had three times less percutaneous permeation of dithranol compared with liposomal formulation (Agarwal et al., 2001). Methotrexate is an antifolate class of anti-neoplastic medicine which is commonly used in the psoriasis therapy. The systemic administration of this drug causes several side effects such as hepatic toxicity. Dermal delivery of methotrexate offers a valuable alternative way to reduce its adverse effects (Javadzadeh et al., 2011). The double-blind placebocontrolled study of methotrexate-loaded niosomal vesicles in chitosan gel on healthy human volunteers and psoriasis patients carried out and its efficacy compared with marketed methotrexate gel. The irritation and skin sensitivity of formulations were assessed via human repeated insult patch test (HRIPT). The HRIPT method did not yield any significant sensitization or irritation on healthy human volunteers. The severity of the lesions of psoriasis was assessed via Psoriasis Area Severity Index (PASI). The global score used to assess the outcome of therapy (on a scale of 0-5), where score of 5 indicates the worsening of lesion and score 0 indicates complete clearance of lesion. There was a three times reduction in PASI scores after 12 weeks of niosomal methotrexate gel. The results of study offer niosomal formulation of methotrexate for dermal treatment of psoriasis due to its better clinical efficacy, tolerability and patient compliance (Lakshmi et al., 2007). Urea is an emollient topically used for the non-surgical removal of dystrophic nails in cases of fungal infections for decades and used as a penetration enhancer for the topical corticosteroid 
treatment of hyperkeratotic psoriatic plaques (Draelos, 2008). Urea may increase ability of skin to retain water due to hygroscopic effect. The same group in another study encapsulated urea into niosomes and prepared a gel using chitosan polymer, to test the same on healthy human volunteers to check the irritation on the skin and to study its clinical effectives on psoriasis patients. Niosomes prepared using span 60 showed a better entrapment than other spans. Better diffusion of drug through the human skin and skin drug deposition were concluded from niosomal gel in comparison to plain gel. The niosomal urea gel and plain gel did not produce any irritation of the human skin. The gels were assessed on psoriasis patients with less than $25 \%$ severity of any types of psoriasis. The niosomal gel showed a significant decrease in the lesion $(p<0.05)$ than plain urea gel. It is suggested that chitosan gel can be used as an adjuvant in the treatment of psoriasis due to antifungal and anti-inflammatory effect of chitosan that supported the action of urea (Lakshmi et al., 2011).

\subsection{Whitening effect}

The attractiveness of pigment-lightening cosmeceuticals comes from the desire to not only fade pigmentation but also to even lighten skin tone (Choi et al., 2006). N-acetyl glucosamine (NAG), which is an amino sugar that occurs widely in nature and essential component of dermal tissues, is well-known for its role as a precursor of hyaluronic acid, a key structural composition of skin. NAG shown to inhibit melanin production in melanocyte culture, thus has a potential to reduce hyperpigmentation by topical administration (Bissett et al., 2007). To improve NAG penetration into the skin Shatalebi et al. encapsulated it into niosomes and investigated its flux across excised rat skin using Franz diffusion cells. All formulations significantly enhanced the drug localization in the skin, as compared to NAG hydroalcoholic solution. Application of negatively charged dicetyl phosphate was suggested the reason for relatively high amount of entrapment of the very water soluble NAG. This study showed the potential of niosomes for improved NAG localization in the skin, as needed in hyperpigmentation disorders (Shatalebi et al., 2010). Ellagic acid, a polyphenol widely found in plants such as pomegranates, inhibits tyrosinase by its copper chelation. It may selectively inhibit melanin synthesis only in UV-activated melanocytes (Choi et al., 2006). A niosomal formulation of ellagic acid was developed from the mixture of Span 60 and Tween 60 for its dermal delivery. Skin distribution study revealed that the ellagic acid-loaded niosomes showed more efficient delivery of ellagic acid through human epidermis and dermis than ellagic acid solution. The results pointed out that the Span 60 and Tween 60 niosomes may be a potential carrier for dermal delivery of ellagic acid (Junyaprasert et al., 2011).

\subsection{Vitiligo}

Vitiligo is an acquired idiopathic, dermatological disorder described by wellcircumscribed milky white macules in which melanocytes in the skin are damaged. Although vitiligo is not a life threatening issue but it might have an important negative 
impaction on the quality of life, even leading to attempted suicide in some cases (Nogueira et al., 2009; Bhawna et al., 2010). Application of novel dermal drug delivery systems can play a key role in vitiligo treatment because side-effects or poor efficacy of conventional topical dosage forms affect their utility and patient compliance (Bhawna et al., 2010). Human tyrosinase gene is responsible for the production of tyrosinase, an enzyme implicates in melanogenesis. The defect of tyrosinase gene is one of the reasons for depigmented skin or vitiligo (Zhang et al., 2005; Kingo et al., 2007). Manosroi et al. prepared elastic cationic niosomes (Tween 61/Cholesterol/ dimethyl dioctadecyl ammonium bromide at 1:1:0.5 molar ratio) for the effective dermal delivery of pMEL34 (tyrosinase encoding plasmid). Percutaneous absorption of formulation was investigated through exercised rat skin by Franz diffusion cells during 6 hours. The flux of pMEL34loaded elastic niosomes was more than non-elastic niosomes in viable epidermis and dermis, while only pMEL34 loaded in elastic cationic noisome was observed in the receiver solution. By application of pMEL34-loaded elastic cationic niosomes in melanoma cell lines showed about four times higher tyrosinase gene expression than the free and the loaded plasmid in nonelastic niosomes means higher tyrosinase activity for efficient topical delivery in vitiligo therapy (Manosroi et al., 2010). In another study, luciferase plasmid (pLuc) was encapsulated in non-elastic and elastic cationic nanovesicles (niosomes and liposomes) and its transdermal absorption was investigated through rat skin follow by stratum corneum stripping or iontophoresis techniques. Free pLuc with or without the stratum corneum stripping and iontophoresis techniques and pLuc-loaded in nonelastic vesicles without the application techniques could not penetrate skin. Though, the elastic vesicles even without any application techniques can increase the transdermal absorption of pLuc. In case of elastic vesicles, the pLuc-loaded niosomes provided higher pLuc flux than that in liposomes. After 6 hours, pLuc encapsulated in nanovesicles with the iontophoresis application was still intact, but the free pLuc was degraded. Result of this study has showed the superior delivery of pLuc thorough skin by loading in niosomes, together with the application of iontophoresis, which can be used as a novel method to deliver genetic materials via dermal administration in gene therapy. Specifically, elastic cationic niosomes appeared to be a more promising approach since no additional equipment is required (Manosroi et al., 2009). Both mentioned studies demonstrated the potential application of elastic cationic niosomes as an efficient topical delivery for the purpose of gene therapy.

\subsection{Cutaneous inflammation}

\subsubsection{Non - steroidal anti-inflammatory drugs (NSAIDs)}

Celecoxib, a selective COX-2 inhibitor and most commonly used drug in the treatment of arthritis was embedded in niosomal gel for the purpose of sustained and site-specific delivery. In vitro, ex-vivo and in vivo studies were carried out through albino rats to compare the efficiency of formulation with carbopol gel as control formulation. Niosomal gel showed 6.5 times higher drug deposition in deep skin layer and muscle compare with carbopol gel indicating better drug localization with niosomal gel. A significant reduction of 
rat paw edema was resulted after administration of niosomal formulation compared to that after application of conventional gel confirming better skin permeation and deposition of celecoxib from niosomes. The authors concluded that niosomal gel formulation has great potential for improving site specific delivery, skin accumulation and prolonging release of celecoxib (Kaur et al., 2007). Multilamellar liposomes and niosomes of aceclofenac, a potent analgesic, anti-pyretic and anti-inflammatory agent were prepared and a comparative study was done between them through evaluation of entrapment efficiency, particle size, shape, differential scanning calorimetry, in vitro drug release and 3 months stability study. Results proved that niosomes have better stability than liposomes. Both vesicular systems showed considerable sustained anti-inflammatory activity compared with the commercial product, however niosomes being superior to liposomes as clearly showed by both oedema rate and inhibition rate percentages assessed by the rat paw oedema technique (Nasr et al., 2008). Proniosomal formulation of gugulipid (anti-inflamatory agent) was fabricated and characterized through in vitro drug release study and invivo anti-inflammatory activity via carrageenan-induced rat hind-paw method. In vitro study of proniosomal gel throught semi-permeable membrane exhibit the initial faster release followed by slow sustained release of gugulipid for $8 \mathrm{~h}$. Gugulipid incoporated proniosomal gel showed good antiinflammatory activity but not as good as commercial product diclofenac (Voveran ${ }^{\circledR}$ Emulgel). The authors state that proniosome formulation improve antiinflammatory activity of gugulipids comparable to topical NSAIDs (Goyal et al., 2011). A niosome based transdermal drug delivery of nimesulide was prepared by lipid film hydration technique using different nonionic surfactants, Tweens ${ }^{\circledR}$ and Spans ${ }^{\circledR}$ and optimized for highest percent drug entrapment. Formulations were extensively characterized and evaluated in-vitro performance followed by in-vivo evaluation in rats by carrageenan induced rat paw edema method. The results were compared with plain drug gel, niosomally entrapment drug in carbopol gel base and marketed formulation. The percentage of edema inhibition was the highest for niosomal nimesulide gel after 24 hours more than five and three times than plain drug gel and market formulation, respectively. The results of this research suggest that niosomal formulation can offer consistent and prolonged anti-inflammatory effect and may improve therapeutic index of the formulation and is also expected to minimize the side effect due to drug localization at the site of action (Shahiwala et al., 2002).

\subsubsection{Glucocorticosteroids}

Topical corticosteroids are administered for the variety of dermatological disorders. Application of corticosteroid in proper carrier may help to prolonged action, subsequently less frequent administration and reduction of adverse effects. In a study, clobetasol propionate, a potent corticosteroid acts as an anti-inflammatory and anti-pruritic, was loaded in niosomal gel and its performance was compare with marketed gel and pure drug in term of in vitro drug release and in vivo pharmacodynamic studies. The results showed that the niosomal gel formulation provided a prolonged action due to the entrapment of clobetasol in vesicles (Lingan et al., 2011). 


\subsubsection{Antihistamines}

Hence antihistamines are frequently administered as first line treatment to reduce the symptoms of urticaria pigmentosa because histamine is the single most important mediator involved (Osvaldo et al., 2010). The conventional oral administration of hydroxyzine hydrochloride (an antihistamine) causes CNS sedation, dry mouth and tachycardia whereas topical use in the form of semisolid dosage forms would lead to systemic side effects (Elzainy et al., 2003). A modified proniosomal gel of hydroxyzine hydrochloride was prepared by coacervation phase separation technique with different combination of nonionic surfactants (Tweens and Spans) with phospholipids. Statistical experimental design was applied to optimize the various formulation variables. The optimized formulations were evaluated in vitro, ex vivo permeation, skin deposition, skin irritation and stability studies. The three months stability at refrigeration temperature and quite high encapsulation efficiency (95\%) and drug deposition in the stratum corneum in $24 \mathrm{~h}(90 \%)$ were found for optimized formulation. The results indicated that modified proniosomal formulations of hydroxyzine hydrochloride were appropriate for topical drug delivery system for the treatment of localized urticarial (Rita et al., 2012). This formulation potentially improves drug penetration into the stratum corneum and localizes the drug within the dermoepidermal layers which would offer prompt onset and prolonged duration of action due to maintenance of effective concentrations in the skin, while systemic serum concentrations would be low (Carafa et al., 1998; Kirjavainen et al., 1999; Elzainy et al., 2003). Chlorpheniramine maleate is one of the most commonly used antihistamines and topically administered for skin disorders such as sunburns, urticaria, angioedema, pruritus, and insect bites. The proniosomes containing Span 40/lecithin/cholestrol formulated by ethanol showed optimum stability, loading efficiency, and particle size and appropriate release kinetic for percutaneous delivery of chlorpheniramine maleate. Ease of preparation and use of proniosomes were introduced as the greatest advantages provided by these types of dosage forms (Varshosaz et al., 2005).

\subsection{Hair loss - Alopecia}

The pilosebaceous unit including sebaceous gland, hair follicle and hair shaft has a unique biochemistry, metabolism and immunology. Targeted drug delivery may improve current therapeutic approaches to treat diseases of follicular origin (Weiner, 1998). In an increasing amount of topical studies, niosomes have been revealed to target drug delivery to the pilosebaceous unit. Androgenic alopecia (male pattern hair loss) is the most common reason of hair loss in men and characterized by the progressive hair thinning in genetically susceptible men (Jung et al., 2006). Semi-purified fraction of Oryza sativa contains the unsaturated fatty acids such as gamma-linolenic acid, linoleic acid and oleic acid have been proved to have anti-hair loss activity by inhibition of 5a-reductase type 1 (a key enzyme of androgenic alopecia) in DU-145 cell lines (Ruksiriwanich et al., 2011). Manosroi et al. prepared cationic niosomes (for higher stability) encapsulated with this extract and investigated physicochemical characteristics and transfollicular penetration of niosomes through porcine skin using follicular closing technique by Franz diffusion cells. The result of 
this study confirmed efficient transfollicular delivery of unsaturated fatty acids using cationic niosomes as well as the advantage of low systemic effect than the neutral niosomes (Manosroi et al., 2012). Thai Lanna medicinal plants have been reported to improve hair growth and reduce hair loss owing to increase the blood circulation, anti-fungal, antibacterial, and anti-oxidation of the hair roots. But, the extracts of these plants are naturally not stable and are problematic to be absorbed through hair follicles. Niosomal formulation containing the Thai Lanna plant extracts, such as turmeric, chili, ginger and Tong-PanChang extract incorporated in gel was fabricated and tested in 20 human volunteers (9 males and 11 females). Results showed a significant increase in hair density and a decrease in hair loss without any irritation in all subjects from the 8-week application $(p<0.01)$ in nonheredity alopecia volunteers, with non-differences in male and female volunteers. This in vivo study confirms potential of niosomes carrier for topical application of Thai Lanna medicinal plants for anti-hair loss (Manosroi et al., 2008). The enzyme 5a-reductase, responsible for production of dihydrotestosterone from testosterone in hair follicles, which is supposed to be the reason of androgenetic alopecia, can be inhibited by finasteride. Finasteride shows several unwanted systemic adverse effects which will be reduced if it acts locally in the hair follicles (Javadzadeh et al., 2010). Tabbakhian et al. studied dermal application of finasteride-containing vesicles (niosomes and liposomes) for increasing drug concentration at the pilosebaceous units, as compared with finasteride hydroalcoholic solution. Both in vitro permeation of $3 \mathrm{H}$-finasteride through hamster flank skin and in vivo deposition studies in hamster ear demonstrated the potentials of liquid-state niosomes and liposomes for successful delivery of finasteride to the pilosebaceous units compared with hydroalcoholic solution while optimized niosomal formulation also showed higher targeting ration than liposomal vesicles (Tabbakhian et al., 2006). Minoxidil, the most commonly used medicine for the treatment of androgenic alopecia with unknown mechanism of action on hair follicles (Messenger et al., 2004) were encapsulated into noisome and liposome vesicles. The percutaneous absorption study was carried out in vitro using vertical diffusion Franz cells using human skin and the results compared with dissolved minoxidil in propylene glycol-water-ethanol solution as a control. Although penetration of niosomal minoxidil in epidermal and dermal layers was greater than control solution but lower than liposomal formulations. These differences suggested to be attributed to the smaller size and the greater potential targeting to skin and skin appendages of liposomal carriers, which enhanced globally the skin drug delivery. This work generally suggests that niosomes and liposomes have a proper potential for drug dermal targeting and could be considered as a reasonable and practicable therapeutic approach to skin diseases such as hair loss (Mura et al., 2007). In a another study the effects of niosomal minoxidil formulation on the drug penetration in hairless mouse skin were investigated by in vitro permeation experiments, and the results compared with control minoxidil hydroalcoholic solution and a leading commercial topical formulation "Minoxyl ${ }^{\mathrm{TM}}$ " containing $5 \%$ minoxidil and $1 \%$ dexpentanol. The result of study showed niosomal formulations increased the percentage of dose accumulated in the skin $(1.03 \pm 0.18$ to $19.41 \pm 4.04 \%)$ compared with control and commercial formulations $(0.48 \pm 0.17$ and $0.11 \pm 0.03$ $\%$, respectively). Physical stability of niosomal formulations which was evaluated for three 
month at refrigerator temperature $\left(2-8^{\circ} \mathrm{C}\right)$ have shown a fairly high retention of minoxidil inside the vesicles $(80 \%)$. The authors finally proposed that these niosomal formulations could be used as a feasible cargo carrier for the dermal delivery of minoxidil and promising approach in treatment alopecia (Balakrishnan et al., 2009). Recently minoxidil-loaded niosomes were prepared with series of surfactant and cholesterol in different molar ratio by ethanol injection method. Surfactant screening exhibited that only Span 20, Span 60 and Tween 20 with cholesterol have ability of nano size vesicle formation and Span 60 was shown to be a better surfactant for niosomal stable form with maximum entrapment efficiency $(31.27 \pm 1.5 \%$ ) and minimal particle size (approx. $219 \mathrm{~nm}$ ). The in vitro skin permeation and deposition study of minoxidil-loaded niosomal gel formulation prepared with 1:2 ratio of Span 60 and cholesterol showed significant improvement in skin accumulation (more than eightfold) as compared with plain minoxidil gel $(17.21 \pm 3.2$ and $2.26 \pm 1.3 \%$, respectively). Result of study demonstrates an increase in cholesterol concentration in niosome vesicles enhance minoxidil skin retention and effect on entrapment efficiency as well as size of niosomes to better cutaneous treatment. It was also concluded that the developed niosomal formulation could be a suitable option for cutaneous targeting of minoxidil (Mali et al., 2012).

\subsection{Acne}

Acne is the most common skin disease of multifactorial origin with an incidence of $70-80 \%$ in adolescence. While topical therapy has an important role in acne treatment, side effects associated with several topical antiacne agents affect their efficacy and patient satisfaction. Niosomes, capable of great features for skin administration, can play an essential role in improving the dermal delivery of antiacne agents by increasing their topical localization with an associated reduction in their adverse effects (Maibach et al., 2005). Benzoyl peroxide is a macrolide antibiotic generally applied for the management of acne either alone or in combination with other antiacne agents. Dermal administration of benzoyl peroxide causes side effects such as skin redness, itching, irritation and edema which lead to discomfort and ignorance of therapy and outcomes in no profit or emergence of resistant to microorganisms. In a study, niosomal benzoyl peroxide incorporated into HPMC gel designed and optimized by partial factorial design. Ex vivo release study on human cadaver skin showed increase in drug skin retention, extended drug release and improved permeation of drug across the skin which in turn will reduce the toxicity of drug and enhance the therapeutic efficacy (Vyas et al., 2011). Gallidermin is mainly promising for the dermal and cosmetic treatment of acne in human medicines because of its greatly active against Propionibacterium acnes and treatment of multidrug resistant Staphylococcus Aureus strains, which is a snowballing problem especially in the hospitals (Kempf et al., 1999). Gallidermin has limited absorption through skin and chemical instability due to its large molecular structure and peptide nature, respectively (Manosroi et al., 2005). Anionic niosomal gallidermin composed of tween 61/cholesterol /dicetylphosphate prepared and antibacterial activity of formulations against Propionibacterium acnes and Staphylococcus Aureus assayed with macrodilution method to determine the minimal concentration of the 
sample necessary to inhibit or kill the microorganisms. Result of study demonstrates gallidermin loaded in niosomes offered lower antibacterial activity against tested microeorganisms compare with unloaded gallidermin due to the niosomes protecting role and making a sustained release of drug. Niosomal furmulation of gallidermin in gel showed more chemically stable at high temperatures and two fold higher cumulative amounts in viable epidermis and dermis of rat skin than aqueous solution of gallidermin. This study suggests that anionic niosomes of gallidermin can be considered as a superior topical antibacterial formulation because of chemical stability and high skin localization with no threat of systemic effect (Manosroi et al., 2010). Trans-retinoic acid or tretinoin, a vitamin A metabolite, is widely used in the topical treatment of various skin diseases such as acne, psoriasis and photoaging. But, high chemical instability and skin irritation strongly limited its administration for topical delivery (Ridolfi et al., 2011). Transdermal delivery of tretinoinloaded niosomal and liposomal formulation was studied through the newborn pig skin using Franz diffusion cells and compared with commercial formulation (Retin $\mathrm{A}^{\circledR}$ ). The effect of charge on the performance niosomes was studied by the preparation of niosomes with either stearylamine and dicetylphosphate as a positive and negative charge inducer, respectively. Small unilamellar negatively charged niosomal formulations saturated with tretinoin showed more upper cutaneous drug retention than both commercial and liposomal formulation. Result of this study demonstrates cutaneous delivery of tretinoin is powerfully affected by vesicle morphology, composition and thermodynamic activity of the drug (Manconi et al., 2006). The ability of niosomes as topical carriers capable of improving the stability of photosensitive drugs was studied by comparing the chemical stability of tretinoin in methanol and in vesicular suspensions exposed both to UV and artificial daylight conditions. Liposomes were also prepared and compared with niosomes. In order to evaluate the influence of vesicle structure on the photostability of tretinoin, tretinoin-loaded vesicles were prepared by the film hydration method, extrusion technique and sonication. Methanol dissolved tretinoin degraded immediately after UV irradiation while the loaded drug into vesicles showed a considerable reduction of the photodegradation process. The photoprotection provided by vesicles varied depending on the vesicle structure and composition. In addition, unilamellar vesicles showed a higher protection of tretinoin than the multilamellar ones. Unilamellar niosomes made from Brij ${ }^{\circledR} 30$ were the formulations with the highest protection of tretinoin (Manconi et al., 2003). In another study, the antiacne activity of the mixture of aromatic volatile oil extracted from Thai medicinal plants enhanced with encapsulating into nano sized niosomal vesicles (Manosroi et al., 2008). Slow penetration of drug through skin is the main disadvantage of transdermal route of delivery. An increase in the penetration rate has been achieved by transdermal delivery of erythromycin incorporated in niosomes for acne therapy (Jayaraman et al., 1996).

\section{Conclusion}

Niosomal drug delivery systems have been demonstrated to be promising controlled drug delivery systems for percutaneous administration. Niosomes also offer successful drug 
localization in skin which are relatively non-toxic and stable. This advantage of niosomes has the potential of strengthening the efficacy of the drug accompanying with reducing its adverse effects associated with drug systemic absorption. Drug-associated challenges such as physical and chemical instability is also can be protected by vesicular carriers. Niosomes appeared to be a well preferred drug delivery system over liposome as niosomes being stable and cost-effective. Hence, many topical drugs may be developed using niosomal systems. But there are still some challenges in this area. Although some new approaches have been developed to overcome the problem of drug loading, it is still remain to be addressed. The researchers should be more alert in the selection of suitable surfactant for noisome preparation due to this fact that the type of surfactant is the main parameter affecting the formation of the vesicles, their toxicity and stability.

\section{Author details}

Yahya Rahimpour

Biotechnology Research Center and Student Research Committee,

Tabriz University of Medical Sciences, Tabriz, Iran

Hamed Hamishehkar

Pharmaceutical Technology Laboratory, Drug Applied Research Center,

Tabriz University of Medical Sciences, Tabriz, Iran

\section{References}

Abd-Elbary, A.; El-laithy, H.M. \& Tadros, M.I., (2008). Sucrose stearate-based proniosomederived niosomes for the nebulisable delivery of cromolyn sodium. Int J Pharm, Vol. 357, No. 1-2, pp. 189-198, ISSN: 0378-5173.

Abdelkader, H.; Ismail, S.; Hussein, A.; Wu, Z.; Al-Kassas, R. \& Alany, R.G., (2012). Conjunctival and corneal tolerability assessment of ocular naltrexone niosomes and their ingredients on the hen's egg chorioallantoic membrane and excised bovine cornea models. Int J Pharm, Vol. 432, No. 1-2, pp. 1-10, ISSN: 0378-5173.

Abdelkader, H.; Wu, Z.; Al-Kassas, R. \& Alany, R.G., (2012). Niosomes and discomes for ocular delivery of naltrexone hydrochloride: morphological, rheological, spreading properties and photo-protective effects. Int J Pharm, Vol. 433, No. 1-2, pp. 142-148, ISSN: 0378-5173.

Agarwal, R.; Katare, O.P. \& Vyas, S.P., (2001). Preparation and in vitro evaluation of liposomal/niosomal delivery systems for antipsoriatic drug dithranol. Int J Pharm, Vol. 228, No. 1, pp. 43-52, ISSN: 0378-5173.

Akhilesh, D.; Hazel, G. \& Kamath, J.V., (2011). Proniosomes - A propitious provesicular drug carrier International Journal of Pharmacy and Pharmaceutical Science Research, Vol. 1, No. 3, pp. 98-103, ISSN: 2249-0337

Azeem, A.; Anwer, M.K. \& Talegaonkar, S., (2009). Niosomes in sustained and targeted drug delivery: some recent advances. J Drug Target, Vol. 17, No. 9, pp. 671-689, ISSN: 1061186X. 
Azmin, M.N.; Florence, A.T. \& Handjani-Vila, R.M., (1985). The effect of non-ionic surfactant vesicle (niosome) entrapment on the absorption and distribution of methotrexate in mice. J Pharm Pharmacol, Vol. 37, No. 4, pp. 237-242, ISSN: 0022-3573

Baillie, A.J.; Coombs, G.H.; Dolan, T.F. \& Laurie, J., (1986). Non-ionic surfactant vesicles, niosomes, as a delivery system for the anti-leishmanial drug, sodium stibogluconate. $J$ Pharm Pharmacol, Vol. 38, No. 7, pp. 502-505, ISSN: 0022-3573.

Balakrishnan, P.; Shanmugam, S.; Lee, W.S.; Lee, W.M.; Kim, J.O.; Oh, D.H.; Kim, D.D.; Kim, J.S.; Yoo, B.K. \& Choi, H.G., (2009). Formulation and in vitro assessment of minoxidil niosomes for enhanced skin delivery. Int J Pharm, Vol. 377, No. 1, pp. 1-8, ISSN: 03785173.

Barry, B.W., (1991). Lipid-Protein-Partitioning theory of skin penetration enhancement. J Control Release, Vol. 15, No. 3, pp. 237-248, ISSN: 0168-3659.

Bayindir, Z.S. \& Yuksel, N., (2010). Characterization of niosomes prepared with various nonionic surfactants for paclitaxel oral delivery. J Pharm Sci, Vol. 99, No. 4, pp. 20492060, ISSN: 1520-6017.

Bhawna, G.; Abir, S.; Amit, B. \& Om, K., (2010). Topical treatment in vitiligo and the potential uses of new drug delivery systems. Indian J Dermatol Venereol Leprol, Vol. 76, ISSN: 0378-6323.

Bissett, D.L.; Robinson, L.R.; Raleigh, P.S.; Miyamoto, K.; Hakozaki, T.; Li, J. \& Kelm, G.R., (2007). Reduction in the appearance of facial hyperpigmentation by topical N-acetyl glucosamine. J Cosmet Dermatol, Vol. 6, No. 1, pp. 20-26, ISSN: 14732165.

Brown, M.B.; Martin, G.P.; Jones, S.A. \& Akomeah, F.K., (2006). Dermal and transdermal drug delivery systems: current and future prospects. Drug Deliv, Vol. 13, No. 3, pp. 175187, ISSN: 1071-7544.

Carafa, M.; Santucci, E.; Alhaique, F.; Coviello, T.; Murtas, E.; Riccieri, F.; Lucania, G. \& Torrisi, M.R., (1998). Preparation and properties of new unilamellar non-ionic/ionic surfactant vesicles. Int J Pharm, Vol. 160, No. 1, pp. 51-59, ISSN: 0378-5173.

Carafa, M.; Santucci, E. \& Lucania, G., (2002). Lidocaine-loaded non-ionic surfactant vesicles: characterization and in vitro permeation studies. Int J Pharm, Vol. 231, No. 1, pp. 21-32, ISSN: 0378-5173.

Cevc, G., (1996). Transfersomes, liposomes and other lipid suspensions on the skin: permeation enhancement, vesicle penetration, and transdermal drug delivery. Crit Rev Ther Drug Carrier Syst, Vol. 13, No. 3-4, pp. 257, ISSN: 0743-4863.

Cevc, G.; Blume, G.; Schätzlein, A.; Gebauer, D. \& Paul, A., (1996). The skin: a pathway for systemic treatment with patches and lipid-based agent carriers. Adv Drug Deliv Rev, Vol. 18, No. 3, pp. 349-378, ISSN: 0169-409X.

Choi, C.M. \& Berson, D.S., (2006). Cosmeceuticals. Semin Cutan Med Surg, Vol. 25, pp.163168, ISSN: 1085-5629

Desai, T.R. \& Finlay, W.H., (2002). Nebulization of niosomal all-trans-retinoic acid: An inexpensive alternative to conventional liposomes. Int J Pharm, Vol. 241, No. 2, pp. 311317, ISSN: 0378-5173. 
Draelos, Z.D., (2008). New channels for old cosmeceuticals: aquaporin modulation. Journal of cosmetic dermatology, Vol. 7, No. 2, pp. 83-83, ISSN: 1473-2165.

Dubey, A.; Prabhu, P. \& Kamath, J., (2012). Nano Structured lipid carriers: A Novel Topical drug delivery system. Int J PharmTech Res, Vol. 4, No. 2, pp. 705-714, ISSN: 09744304.

Dubey, V.; Mishra, D.; Dutta, T.; Nahar, M.; Saraf, D.K. \& Jain, N.K., (2007). Dermal and transdermal delivery of an anti-psoriatic agent via ethanolic liposomes. J Control Release, Vol. 123, No. 2, pp. 148-154, ISSN: 0168-3659.

Elzainy, A.A.W.; Gu, X.; Simons, F.E.R. \& Simons, K.J., (2003). Hydroxyzine from topical phospholipid liposomal formulations: Evaluation of peripheral antihistaminic activity and systemic absorption in a rabbit model. The AAPS Journal, Vol. 5, No. 4, pp. 4148 ,

Fang, J.Y.; Hong, C.T.; Chiu, W.T. \& Wang, Y.Y., (2001). Effect of liposomes and niosomes on skin permeation of enoxacin. Int J Pharm, Vol. 219, No. 1-2, pp. 61-72, ISSN: 03785173.

Firthouse, P.U.M.; Halith, S.M.; Wahab, S.U.; Sirajudeen, M. \& Mohideen, S.K., (2011). Formulation and evaluation of miconazole niosomes. Int J PharmTech Res, Vol. 3, No. 2, pp. 1019-1022, ISSN: 0974-4304.

Gašperlin, M. \& Gosenca, M., (2011). Main approaches for delivering antioxidant vitamins through the skin to prevent skin ageing. Expert Opin Drug Del, Vol. 8, No. 7, pp. 905-919, ISSN: $1742-5247$.

Ghafourian, T.; Zandasrar, P.; Hamishekar, H. \& Nokhodchi, A., (2004). The effect of penetration enhancers on drug delivery through skin: a QSAR study. J Control Release, Vol. 99, No. 1, pp. 113-125, ISSN: 0168-3659.

Gopinath, D.; Ravi, D.; Rao, B.R.; Apte, S.S.; Renuka, D. \& Rambhau, D., (2004). Ascorbyl palmitate vesicles (Aspasomes): formation, characterization and applications. Int $J$ Pharm, Vol. 271, No. 1-2, pp. 95-113, ISSN: 0378-5173.

Goyal, C.; Ahuja, M. \& Sharma, S.K., (2011). Preparation and evaluation of antiinflammatory activity of gugulipid-loaded proniosomal gel. Acta Pol Pharm, Vol. 68, No. 1, pp. 147-150, ISSN: 0001-6837.

Handjani-Vila, R.M.; Ribier, A.; Rondot, B. \& Vanlerberghe, G., (1979). Dispersions of lamellar phases of non-ionic lipids in cosmetic products. Int J Cosmetic Sci, Vol. 1, pp. 303-314,

Higaki, K.; Nakayama, K.; Suyama, T.; Amnuaikit, C.; Ogawara, K. \& Kimura, T., (2005). Enhancement of topical delivery of drugs via direct penetration by reducing blood flow rate in skin. Int J Pharm, Vol. 288, No. 2, pp. 227-233, ISSN: 0378-5173.

Hofland, H.; Bowuwstra, J.A.; Ponec, M. Boddé, H.E.; Spies, F.; Coos Verhoef, J.; Junginger H.E., (1991). Interactions of non-ionic surfactant vesicles with cultured keratinocytes and human skin in vitro: a survey of toxicological aspects and ultrastructural changes in stratum corneum. J Control Release, Vol. 16, No. 1, pp. 155-167, ISSN: 0168-3659. 
Hofland, H.E.J.; Bouwstra, J.A.; Spies, F. \& Bodde, H.E., (1992). Safety aspect of nonionic surfactant vesicles- a toxicity study related to the physicochemical characteristics of nonionic surfactants. J Pharm Pharmacol, Vol. 44, pp. 287-292, ISSN: 0022-3573

Hofland, H.E.J.; van der Geest, R.; Bodde, H.E.; Junginger, H.E. \& Bouwstra, J.A., (1994). Estradiol permeation from nonionic surfactant vesicles through human stratum corneum in vitro. Pharm Res, Vol. 11, No. 5, pp. 659-664, ISSN: 07248741.

Hu, C. \& Rhodes, D.G., (2000). Proniosomes: a novel drug carrier preparation. Int J Pharm Vol. 206, pp. 110-122, ISSN: 0378-5173.

Jain, C.P.; Vyas, S.P. \& Dixit, V.K., (2006). Niosomal system for delivery of rifampicin to lymphatics. Indian J Pharm Sci, Vol. 68, No. 5, pp. 575, ISSN: 0250-474X.

Javadzadeh, Y. \& Hamishehkar, H., (2011). Enhancing percutaneous delivery of methotrexate using different types of surfactants. Colloid Surface B, Vol. 82, No. 2, pp. 422-426, ISSN: 0927-7765.

Javadzadeh, Y.; Shokri, J.; Hallaj-Nezhadi, S.; Hamishehkar, H. \& Nokhodchi, A., (2010). Enhancement of percutaneous absorption of Finasteride by cosolvents, cosurfactant and surfactants. Pharm Dev Technol, Vol. 15, No. 6, pp. 619-625, ISSN: 1083-7450.

Jayaraman, S.C.; Ramachandran, C. \& Weiner, N., (1996). Topical delivery of erythromycin from various formulations: An in vivo hairless mouse study. J Pharm Sci, Vol. 85, No. 10, pp. 1082-1084, ISSN: 1520-6017.

Jung, S.; Otberg, N.; Thiede, G.; Richter, H.; Sterry, W.; Panzner, S. \& Lademann, J., (2006). Innovative liposomes as a transfollicular drug delivery system: penetration into porcine hair follicles. J Invest Dermatol, Vol. 126, No. 8, pp. 1728-1732, ISSN: 0022-202X.

Junyaprasert, V.B.; Singhsa, P.; Suksiriworapong, J. \& Chantasart, D., (2011). Physicochemical properties and skin permeation of span 60/tween 60 niosomes of ellagic acid. Int J Pharm, Vol. doi:10.1016/j.ijpharm.2011.11.032, ISSN: 03785173.

Kaur, K.; Jain, S.; Sapra, B. \& Tiwary, A.K., (2007). Niosomal gel for site-specific sustained delivery of anti-arthritic drug: in vitro-in vivo evaluation. Curr Drug Deliv, Vol. 4, No. 4, pp. 276-282, ISSN: 1567-2018.

Kempf, M.; Theobald, U. \& Fiedler, H.P., (1999). Economic improvement of the fermentative production of gallidermin by Staphylococcus gallinarum. Biotechnol lett, Vol. 21, No. 8, pp. 663-667, ISSN: 0141-5492.

Khan, A.; Sharma, P.K.; Visht, S. \& Malviya, R., (2011). Niosomes as colloidal drug delivery system: A review. Journal of Chronotherapy and Drug Delivery, Vol. 2, No. 1, pp. 15-21,

Kingo, K.; Aunin, E.; Karelson, M.; Philips, M.A.; Rätsep, R.; Silm, H.; Vasar, E.; Soomets, U. \& Köks, S., (2007). Gene expression analysis of melanocortin system in vitiligo. J Dermatol Sci, Vol. 48, No. 2, pp. 113-122, ISSN: 0923-1811.

Kirjavainen, M.; Mönkkönen, J.; Saukkosaari, M.; Valjakka-Koskela, R.; Kiesvaara, J. \& Urtti, A., (1999). Phospholipids affect stratum corneum lipid bilayer fluidity and drug 
partitioning into the bilayers. J Control Release, Vol. 58, No. 2, pp. 207-214, ISSN: 01683659.

Kumar, G.P. \& Rao, P.R., (2012). Ultra deformable niosomes for improved transdermal drug delivery: The future scenario. Asian Journal of Pharmaceutical Sciences, Vol. 7, No. 2, pp. 96-109, ISSN: 0974-2441

Lakshmi, P.K. \& Bhaskaran, S., (2011). Phase II study of topical niosomal urea gel - an adjuvant in the treatment of psoriasis. International Journal of Pharmaceutical Sciences Review and Research, Vol. 7, No. 1, pp. 1-7, ISSN: 0976 -044X

Lakshmi, P.K.; Devi, G.S.; Bhaskaran, S. \& Sacchidanand, S., (2007). Niosomal methotrexate gel in the treatment of localized psoriasis: Phase I and phase II studies. Indian J Dermatol Venereol Leprol, Vol. 73, No. 3, pp. 157, ISSN: 0378-6323.

Lala, S.; Pramanick, S.; Mukhopadhyay, S.; Bandyopadhyay, S. \& Basu, M.K., (2004). Harmine: Evaluation of its antileishmanial properties in various vesicular delivery systems. J Drug Target, Vol. 12, No. 3, pp. 165-175, ISSN: 1061-186X.

Lingan, M.A.; Sathali, A.A.H.; Kumar, M.R.V. \& Gokila, A., (2011). Formulation and evaluation of topical drug delivery system containing clobetasol propionate niosomes. Sci Revs Chem Commun, Vol. 1, No. 1, pp. 7-17,

Maibach, H.I. \& Choi, M.J., (2005). Liposomes and niosomes as topical drug delivery systems. Skin Pharmacol Physiol, Vol. 18, pp. 209-219, ISSN: 1660-5527.

Malhotra, M. \& Jain, N.K., (1994). Niosomes as drug carriers. Ind Drugs, Vol. 31, No. 3, pp. 81-86,

Mali, N.; Darandale, S. \& Vavia, P., (2012). Niosomes as a vesicular carrier for topical administration of minoxidil: formulation and in vitro assessment. Drug Deliv Transl Res, pp. 1-6, ISSN: 2190-393X.

Manconi, M.; Sinico, C.; Valenti, D.; Lai, F. \& Fadda, A.M., (2006). Niosomes as carriers for tretinoin: III. A study into the in vitro cutaneous delivery of vesicle-incorporated tretinoin. Int J Pharm, Vol. 311, No. 1-2, pp. 11-19, ISSN: 0378-5173.

Manconi, M.; Valenti, D.; Sinico, C.; Lai, F.; Loy, G. \& Fadda, A.M., (2003). Niosomes as carriers for tretinoin: II. Influence of vesicular incorporation on tretinoin photostability. Int J Pharm, Vol. 260, No. 2, pp. 261-272, ISSN: 0378-5173.

Manosroi, A.; Khanrin, P.; Lohcharoenkal, W.; Werner, R.G.; Götz, F.; Manosroi, W. \& Manosroi, J., (2010). Transdermal absorption enhancement through rat skin of gallidermin loaded in niosomes. Int J Pharm, Vol. 392, No. 1, pp. 304-310, ISSN: 03785173.

Manosroi, A.; Khositsuntiwong, N.; Götz, F.; Werner, R.G. \& Manosroi, J., (2009). Transdermal enhancement through rat skin of luciferase plasmid DNA loaded in elastic nanovesicles. J Liposome Res, Vol. 19, No. 2, pp. 91-98, ISSN: 0898-2104.

Manosroi, A.; Ruksiriwanich, W.; Abe, M.; Manosroi, W. \& Manosroi, J., (2012). Transfollicular enhancement of gel containing cationic niosomes loaded with unsaturated fatty acids in rice (Oryza sativa) bran semi-purified fraction. Eur J Pharm Biopharm, Vol. 81, No. 2, pp. 303-313, ISSN: 0939-6411. 
Manosroi, A.; Sritapunya, T.; Jainonthee, P. \& Manosroi, J., (2008). Anti P. acne activity of cream containing aromatic volatile oil from Thai medicinal plants entrapped in niosomes for acne treatment.

Manosroi, A.; Wongtrakul, P.; Manosroi, J.; Midorikawa, U.; Hanyu, Y.; Yuasa, M.; Sugawara, F.; Sakai, H. \& Abe, M., (2005). The entrapment of kojic oleate in bilayer vesicles. Int J Pharm, Vol. 298, No. 1, pp. 13-25, ISSN: 0378-5173.

Manosroi, J.; Khositsuntiwong, N. \& Manosroi, A., (2008). Performance test of gel containing extracts from Thai Lanna medicinal plants entrapped in niosomes for hair loss treatment.

Manosroi, J.; Khositsuntiwong, N.; Manosroi, W.; Götz, F.; Werner, R.G. \& Manosroi, A., (2010). Enhancement of transdermal absorption, gene expression and stability of tyrosinase plasmid (pMEL34)-loaded elastic cationic niosomes: Potential application in vitiligo treatment. J Pharm Sci, Vol. 99, No. 8, pp. 3533-3541, ISSN: 15206017.

Messenger, A. \& Rundegren, J., (2004). Minoxidil: mechanisms of action on hair growth. Brit J Dermatol, Vol. 150, No. 2, pp. 186-194, ISSN: 0007-0963.

Mura, S.; Pirot, F.; Manconi, M.; Falson, F. \& Fadda, A.M., (2007). Liposomes and niosomes as potential carriers for dermal delivery of minoxidil. J Drug Target, Vol. 15, No. 2, pp. 101-108, ISSN: 1061-186X.

Nasr, M.; Mansour, S.; Mortada, N.D. \& Elshamy, A.A., (2008). Vesicular aceclofenac systems: A comparative study between liposomes and niosomes. J Microencapsul, Vol. 25, No. 7, pp. 499-512, ISSN: 0265-2048.

Nogueira, L.S.; Zancanaro, P.C. \& Azambuja, R.D., (2009). [Vitiligo and emotions]. An Bras Dermatol, Vol. 84, No. 1, pp. 41-45, ISSN: 1806-4841

Nounou, M.M.; El-Khordagui, L.K.; Khalafallah, N.A. \& Khalil, S.A., (2008). Liposomal formulation for dermal and transdermal drug delivery: past, present and future. Recent Pat Drug Deliv Formul, Vol. 2, No. 1, pp. 9-18, ISSN: 1872-2113.

Ogiso, T.; Niinaka, N. \& Iwaki, M., (1996). Mechanism for enhancement effect of lipid disperse system on percutaneous absorption. J Pharm Sci, Vol. 85, pp. 57-64, ISSN: 15206017.

Osvaldo, C.; Duarte, A.F.; Paula, Q.; Rosa, A. \& Luis, D., (2010). Cutaneous mastocytosis: Two pediatric cases treated with topical pimecrolimus. Dermatol Online J, Vol. 16, No. 5, pp. 8, ISSN: 1087-2108

Paolino, D.; Muzzalupo, R.; Ricciardi, A.; Celia, C.; Picci, N. \& Fresta, M., (2007). In vitro and in vivo evaluation of Bola-surfactant containing niosomes for transdermal delivery. Biomed Microdevices, Vol. 9, No. 4, pp. 421-433, ISSN: 1387-2176.

Pardakhty, A.; Varshosaz, J. \& Roulholamini, A., (2007). In vitro study of polyoxyethylene alkyl ether niosomes for delivery of insulin. Int J Pharm, Vol. 328, pp. 130-141, ISSN: 0378-5173.

Parthasarathi, G.; Udupa, N.; Umadevi, P. \& Pillai, G.K., (1994). Niosome encapsulated of vincristine sulfate: Improved anticancer activity with reduced toxicity in mice. J Drug Target, Vol. 2, No. 2, pp. 173-182, ISSN: 1061-186X. 
Perrett, S.; Golding, M. \& Williams, W.P., (1991). A simple method for the preparation of liposomes for pharmaceutical application and characterization of liposomes. J Pharm Pharmacol, Vol. 43, pp. 154-161, ISSN: 2042-7158.

Pugeat, M.; Nicolas, M.H.; Dechaud, H. \& Elmidani, M., (1991). Combination of cyproterone acetate and natural estrogens in the treatment of hirsutism]. J Gynecol Obst Bior R, Vol. 20, No. 8, pp. 1057, ISSN: 0368-2315.

Puglia, C. \& Bonina, F., (2012). Lipid nanoparticles as novel delivery systems for cosmetics and dermal pharmaceuticals. Expert Opin Drug Del, Vol. 9, No. 4, pp. 429-441, ISSN: $1742-5247$.

Rahimpour, Y. \& Hamishehkar, H., (2012). Liposomes in cosmeceutics. Expert Opin Drug Del, Vol. 9, No. 4, pp. 443-455, ISSN: 1742-5247.

Raja Naresh, R.A.; Pillai, G.K.; Udupa, N. \& Chandrashekar, G., (1994). Antiinflammatory activity of niosome encapsulated diclofenac sodium in arthitic rats. Indian J Pharmacol, Vol. 26, pp. 46-48, ISSN: 0253-7613.

Rajera, R.; Nagpal, K.; Singh, S.K. \& Mishra, D.N., (2011). Niosomes: A controlled and novel drug delivery system. Bio Pharm Bull, Vol. 34, No. 7, pp. 945-953, ISSN: 09186158.

Ridolfi, D.M.; Marcato, P.D.; Justo, G.Z.; Cordi, L.; Machado, D. \& Durán, N., (2011). Chitosan-solid lipid nanoparticles as carriers for topical delivery of Tretinoin. Colloid Surface B, ISSN: 0927-7765.

Rita, B. \& Lakshmi, P.K., (2012). Preparation and evaluation of modified proniosomal gel for localised urticaria and optimisation by statistical method. Journal of Applied Pharmaceutical Science, Vol. 2, No. 03, pp. 85-91,

Rogerson, A.; Cummings, J. \& Florence, A.T., (1987). Adriamycin-loaded niosomes: drug entrapment, stability and release. J Microencapsul, Vol. 4, No. 4, pp. 321-328, ISSN: 02652048.

Rubio, L.; Alonso, C.; López, O.; Rodríguez, G.; Coderch, L.; Notario, J.; de la Maza, A. \& Parra, J.L., (2011). Barrier function of intact and impaired skin: percutaneous penetration of caffeine and salicylic acid. Int J Dermatol, Vol. 50, No. 7, pp. 881-889, ISSN: 1365-4632.

Ruckmani, K.; Jayakar, B. \& Ghosal, S.K., (2000). Nonionic surfactant vesicles (niosomes) of cytarabine hydrochloride for effective treatment of leukemias: Encapsulation, storage, and in vitro release. Drug Dev Ind Pharm, Vol. 26, No. 2, pp. 217-222, ISSN: 03639045.

Ruksiriwanich, W.; Manosroi, J.; Abe, M.; Manosroi, W. \& Manosroi, A., (2011). 5 [alpha]Reductase type 1 inhibition of Oryza sativa bran extract prepared by supercritical carbon dioxide fluid. J Supercrit Fluid, ISSN: 0896-8446.

Sahin, N.O. Niosomes as nanocarrier systems. Nanomaterials and nanosystems for biomedical applications2007. p. 67-81.

Sathali, A.A.H. \& Rajalakshmi, G., (2010). Evaluation of transdermal targeted niosomal drug delivery of terbinafine hydrochloride. Int J PharmTech Res, Vol. 2, No. 3, pp. 2081-2089, ISSN: 0974-4304. 
Schreier, H. \& Bouwstra, J., (1994). Liposomes and niosomes as topical drug carriers: dermal and transdermal drug delivery. J Control Release, Vol. 30, No. 1, pp. 1-15, ISSN: 01683659.

Shahiwala, A. \& Misra, A., (2002). Studies in topical application of niosomally entrapped nimesulide. J Pharm Pharm Sci, Vol. 5, No. 3, pp. 220, ISSN: 1482-1826.

Shatalebi, M.A.; Mostafavi, S.A. \& Moghaddas, A., (2010). Niosome as a drug carrier for topical delivery of N-acetyl glucosamine. Res Pharm Sci, Vol. 5, No. 2, pp. 107, ISSN: 1735-5362.

Shi, B.; Fang, C.; You, M.X. \& Pei, Y.Y., (2005). Influence of PEG chain length on in vitro drug release and in vivo pharmacokinetics of hydroxycamptothecin (HCPT) loaded PEG-PHDCA niosomes. Chinese Pharmaceutical Journal, Vol. 40, No. 21, pp. 16431646 ,

Su, Y.H. \& Fang, J.Y., (2008). Drug delivery and formulations for the topical treatment of psoriasis. Expert Opin Drug Del, Vol. 5, No. 2, pp. 235-249, ISSN: 1742-5247.

Tabbakhian, M.; Tavakoli, N.; Jaafari, M.R. \& Daneshamouz, S., (2006). Enhancement of follicular delivery of finasteride by liposomes and niosomes:: 1 . In vitro permeation and in vivo deposition studies using hamster flank and ear models. Int J Pharm, Vol. 323, No. 1-2, pp. 1-10, ISSN: 0378-5173.

Uchegbu, I.F. \& Duncan, R., (1997). Niosomes containing N-(2-hydroxypropyl) methacrylamide copolymer-doxorubicin (PK1): effect of method of preparation and choice of surfactant on niosome characteristics and a preliminary study of body distribution. Int J Pharm, Vol. 155, No. 1, pp. 7-17, ISSN: 0378-5173.

Varshosaz, J.; Pardakhty, A.; Mohsen, S. \& Baharanchi, H., (2005). Sorbitan monopalmitatebased proniosomes for transdermal delivery of chlorpheniramine maleate. Drug Deliv, Vol. 12, No. 2, pp. 75-82, ISSN: 1071-7544.

Venuganti, V.V. \& Perumal, O.P. Nanosystems for dermal and transdermal drug delivery. In: Pathak Y, Thassu D, editors. Drug delivery nanoparticles formulation and characterization. New York: Informa Healthcare USA, Inc; 2009. p. 126-155.

Verma, A.K. \& Bindal, M., (2012). A review on niosomes: an ultimate controlled and novel drug delivery carrier. Int J Nanoparticles Vol. 5, No. 1, pp. 73-87,

Vyas, J.; Vyas, P.; Raval, D. \& Paghdar, P., (2011). Development of topical niosomal gel of benzoyl peroxide. ISRN Nanotechnology, Vol. 2011, ISSN: 2090-6064.

Weiner, N., (1998). Targeted follicular delivery of macromolecules via liposomes. Int J Pharm, Vol. 162, No. 1-2, pp. 29-38, ISSN: 0378-5173.

Yadav, J.D.; Kulkarni, P.R.; Vaidya, K.A. \& Shelke, G.T., (2011). Niosomes: a review. J Pharm Res, Vol. 4, pp. 632-636, ISSN: 0974-6943.

Yoshida, H.; Lehr, C.M.; Kok, W.; Junginger, H.E.; Verhoef, J.C. \& Bouwstra, J.A., (1992). Niosomes for oral delivery of peptide drugs. J Control Release, Vol. 21, No. 1-3, pp. 145153, ISSN: 0168-3659.

Yoshioka, T.; Sternberg, M.; Moody, M. \& Florence, A.T., (1992). Niosomes from Span surfactants: Relations between structure and form. J Pharm Pharmacol, Vol. 44, pp. 10441044, ISSN: 0022-3573. 
Zhang, X.J.; Chen, J.J. \& Liu, J.B., (2005). The genetic concept of vitiligo. J Dermatol Sci, Vol. 39, No. 3, pp. 137-146, ISSN: 0923-1811. 